\title{
SEGGERN, Harm von, FOUQUET, Gerhard, Adel und Zahl. Studien zum adligen Rechnen und Haushalten in Spätmittelalter und früher Neuzeit
}

Joseph Morsel

\section{OpenEdition}

\section{Journals}

Édition électronique

URL : http://journals.openedition.org/ifha/1007

DOI : 10.4000/ifha.1007

ISSN : 2198-8943

Éditeur

IFRA - Institut franco-allemand (sciences historiques et sociales)

Référence électronique

Joseph Morsel, «SEGGERN, Harm von, FOUQUET, Gerhard, Adel und Zahl. Studien zum adligen Rechnen und Haushalten in Spätmittelalter und früher Neuzeit », Revue de l'IFHA [En ligne], Date de recension, mis en ligne le 01 janvier 2004, consulté le 22 septembre 2020. URL : http://journals.openedition.org/ifha/ 1007 ; DOI : https://doi.org/10.4000/ifha.1007

Ce document a été généré automatiquement le 22 septembre 2020.

(C)IFHA 


\title{
SEGGERN, Harm von, FOUQUET, Gerhard, Adel und Zahl. Studien zum adligen Rechnen und Haushalten in Spätmittelalter und früher Neuzeit
}

\author{
Joseph Morsel
}

Dans une perspective d'histoire des idées, l'apparition de sources comptables est souvent reliée à l'avènement d'une nouvelle rationalité (voire de la rationalité tout court), dont témoignerait ne serait-ce que la diffusion considérable de l'écrit à la fin du Moyen Âge (le XIIIe s. marquant justement et l'apparition des comptabilité et l'explosion numérique de la scripturalité). L'esprit comptable (voire la rationalité) est aussi classiquement associé à l'esprit bourgeois, et donc opposé à l'esprit noble, censé dépenser sans compter. Mais depuis quelques temps, dans le cadre ou à la suite des travaux menés autour de W. Paravicini sur les cours princières, il s'est avéré que le fait de compter n'était pas propre à la seule bourgeoisie marchande mais aussi aux administrations princières. Les sources comptables doivent ainsi être considérées comme des instruments d'organisation de la « maison » en question, et passent donc du statut d'expression de la rationalité bourgeoise à celui d'instrument de domestication, elle-même forme initiale de la rationalité étatique (pour ne pas parler de raison d'État) qui a engendré la "statistique ». Que compter soit une pratique relativement étrangère à la noblesse (le titre du volume en question, "Noblesse et nombre ", sous-entendant une bipolarité) doit donc être réexaminé dans une perspective autre que celle du simple sous-développement intellectuel. C'est ce à quoi entendent contribuer les 15 textes du volume en question : un texte d'introduction, 13 études de cas et une conclusion, suivie d'une bibliographie sélective concernant les comptabilités aristocratiques qui, à elle seule, rend l'ouvrage très utile. Les études de cas concernent principalement l'Empire (duché de Saxe-Lunebourg, landgraviat de Hesse et comté d'Isenburg, baronnie de Weinsberg, évêché de Bâle, comté de Nassau, comté de Hollande, soldats allemands en Italie, Bavière), deux portent sur la Bourgogne et une sur l'Angleterre. Toutes portent sur le XIVe et/ou le XVe s., avec parfois une 
prolongation sur le XVIe s., à l'exception de la contribution bavaroise (1763). Les sources comptables traitées permettent certes de reconstruire les systèmes financiers qui leur ont donné naissance (notamment les systèmes de collecte des recettes et d'assignation des paiements, c'est-à-dire aussi le recours variable aux monnaies), mais elles sont surtout utilisées soit pour reconstituer le quotidien (la consommation) des aristocrates concernés ou encore de leurs serviteurs plus ou moins occasionnels, soit pour établir, justement, une sociographie de leur entourage (sous forme de listes ou, plus élaborée, de clusters). Les sources s'étendant sur de longues périodes, comme en Bourgogne, sont aussi utilisables pour étudier les prix.

2 La lecture de cet ouvrage est ainsi particulièrement instructive. On pourra cependant regretter l'absence de réflexion sur le sens social du dénombrement, et notamment sur les effets sociaux. Car l'ensemble des travaux repose sur l'idée implicite que le dénombrement revient à quantifier (et aussi, pour certains, à classer) des choses préexistantes, ce qui est rien moins qu'évident : dénombrer n'est jamais un simple acte descriptif, il est aussi toujours un acte prescriptif, non seulement classificateur mais aussi sociogénétique (cf. les travaux d'A. Desrosières, notamment La politique des grands nombres, 1993), d'autant que les nombres étaient eux-mêmes pris dans une logique sociale interdisant de réduire leur usage à une simple quantification (cf. les travaux de G. Beaujouan). Compter (qui plus est par écrit) ne renvoie ainsi pas à un simple acte d'inventaire quantifié mais relève d'entreprises (évidemment inconscientes) de construction sociale - ce qui place l'historien devant le problème du respect ou non de l'organisation des comptes : comme le montre bien le volume en question, les sources comptables sont le plus souvent construites de manière extrêmement variable, ce qui non seulement rend difficile la reconstitution et la comparaison des budgets, mais en outre devrait interroger la pertinence qu'il y a à le faire, au-delà de la simple approche comptable à laquelle se restreint l'ouvrage. Que compter soit une pratique non inhérente à la noblesse prendrait ainsi un autre sens : si l'on n'y compte pas, ce n'est pas par absence de rationalité, mais en vertu d'un autre mode d'organisation sociale, dans laquelle la notion de budget (Haushalt) n'a que faire. C'est là que le rapport signalé plus haut entre ces comptabilités et l'organisation de la " maison " peut fournir une piste, surtout si l'on considère également que les sources fiscales ne sont jamais de simples relevés, mais qu'elles imposent fiscalement des unités de mesure sociales - en l'occurrence, précisément, celle du ménage (également Haushalt), du feu, de la famille. L'avènement de la comptabilité serait ainsi moins un signe de rationalisation des pratiques économiques que de transformation des pratiques sociales - et le rapport entre noblesse et nombre devrait donc être considéré sous cet angle, l'avènement du dénombrement y réalisant (pour eux) et y signalant (pour nous) l'avènement de la " maison ». En tout cas, il s'agit bien d'un volume à connaître.

4 Universitaire de France) 